\section{Original Article}

Ann Liver Transplant 2021;1(2):135-139

https://doi.org/10.52604/alt.21.0026

Check for updates
ALT

pISSN 2765-5121

elSSN 2765-6098

\title{
Analysis of nationwide volume of liver transplantation in Korea during 2020 shows no definitive decrease during COVID-19 outbreak
}

\author{
Yong-Kyu Chung ${ }^{1}$, Sung-Hwa Kang ${ }^{2}$ \\ 1 Department of Surgery, Haeundae Paik Hospital, Inje University College of Medicine \\ ${ }^{2}$ Department of Surgery, Dong-A University Hospital, College of Medicine Dong-A University, Busan, Korea
}

Received October 7, 2021

Revised October 19, 2021

Accepted October 28, 2021

Published online November 4, 2021

Corresponding author: Yong-Kyu Chung Department of Surgery, Haeundae Paik Hospital, Inje University College of Medicine, Haewoondaero 875, Haewoondae-gu, Busan 48108, Korea

E-mail: iteacher13@gmail.com

https://orcid.org/0000-0002-2132-2450

() The Korean Liver Transplantation Society (c) (i) (s) This is an Open Access article distributed under the terms of the Creative Commons Attribution Non-Commercial License (http://creativecommons.org/licenses/by-nc/4.0/) which permits unrestricted non-commercial use, distribution, and reproduction in any medium, provided the original work is properly cited.
Background: Recent outbreak of Coronavirus disease 2019 (COVID-19) has discouraged organ donation. To determine its impact on liver transplantation (LT) at Korean transplant centers, it is necessary to analyze the incidence and type of LT in each center.

Methods: The number of deceased donor LT (DDLT) and living donor LT (LDLT) operations performed during the year 2020 were analyzed using the Korean Network for Organ Sharing (KONOS) database.

Results: During the 21-year period from 2000 to 2020, the total number of all deceased organ donors was 6,064 and 5,252 cases of DDLT were performed. The deceased donor and DDLT case numbers were 450 and 391 , respectively, in the year 2019 , and 478 and 395 , respectively, in the year $2020(p=0.284)$. The mean number of monthly DDLT was $32.6 \pm 5.7$ in 2019 and $32.9 \pm 4.7$ in 2020 ( $p=0.877)$. The case numbers of DDLT and LDLT in 2020 were 395 and 1,221, respectively. Forty-one centers performed both DDLT and LDLT, but five centers performed only DDLT and another 10 centers performed only LDLT. The five major LT centers performed 974 cases, which accounted for $60.3 \%$ of nation-wide LT volume in 2020 , including $45.3 \%$ of DDLT and $65.1 \%$ of LDLT.

Conclusion: The results of this study suggested that national activities of DDLT and LDLT remained stable in Korea in the year 2020, despite of the ongoing COVID-19 pandemic. Further follow-up studies are necessary to validate the results of this study.

Keywords: Coronavirus disease 19; Deceased donor; Living donor; Immunosuppression; Donation promotion

\section{INTRODUCTION}

Liver transplantation (LT) is an effective treatment for patients with end-stage liver disease because of improved outcomes and broadening of indications. The shortage of deceased organ donors and increased demand for LT have increased the liver grafts available for LT. Living donor liver transplantation (LDLT) has been settled as the primary type 
of LT in Korea for the last 20 years [1-3].

Because of gradual increase in the number of deceased donors as well as accumulation of institutional experience related to LDLT [4], the majority of university hospitals and a few private hospitals in Korea implement their own LT program for deceased donor liver transplantation (DDLT) or LDLT, or both. The number of hospitals performing LT has increased gradually to 56 centers in the year 2020 .

The recent outbreak of Coronavirus disease 2019 (COVID-19) has discouraged organ donation [5-7]. To determine its real-world impact on the LT volume in Korean transplant centers, it is necessary to analyze the number and type of LT performed at each center. We herein present the number of DDLT and LDLT operations during the year 2020 in 56 LT centers using the Korean Network for Organ Sharing (KONOS) database.

\section{PATIENTS AND METHODS}

The purpose of this study was to determine the annual number of DDLT and LDLT performed at individual LT centers in a single year of 2020 . This study consists of two components. The first component includes an analysis of the annual number of DDLT and LDLT performed at the Korean LT centers, using the KONOS database. The annual LT volume in $\mathbf{2 0 2 0}$ was compared with the previous data. The second component includes an assessment of recipient profiles according to their age at LT and sex using the KONOS database. We defined pediatric LT based on recipient's age $\leq 18$ years and adult LT based on recipient's age $\geq 19$ years. The study protocol was waived for approval be-

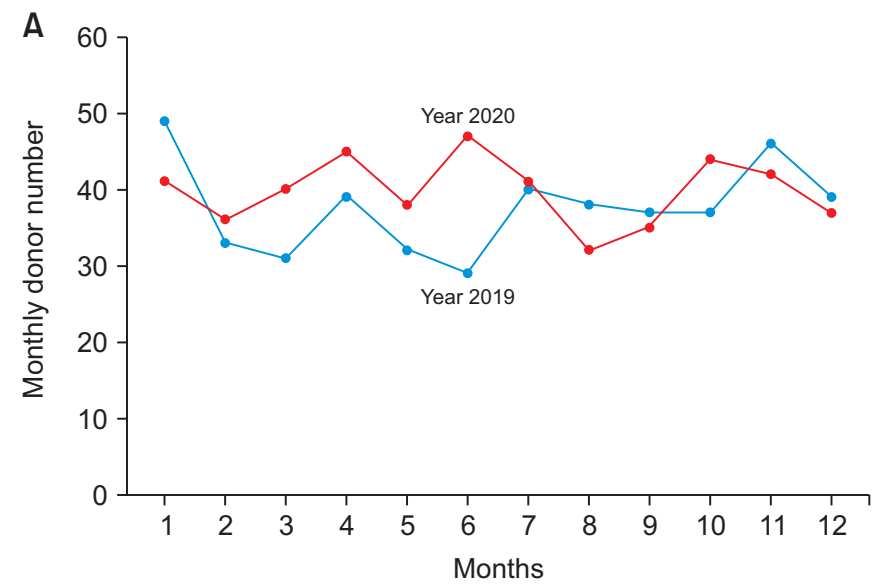

cause of this study used only publicly open data. This study was performed in accordance with the ethical guidelines of the World Medical Association Declaration of Helsinki 2013.

A chi-square test was used for comparison of the number of LTs. A p-value $<0.05$ was considered statistically significant. All statistical analyses were performed using SPSS version 22 (IBM Co., Armonk, NY, USA).

\section{RESULTS}

\section{Comparison of Annual Numbers of Deceased Donors and DDLT Operations}

During the 21-year period from 2000 to 2020 , the total

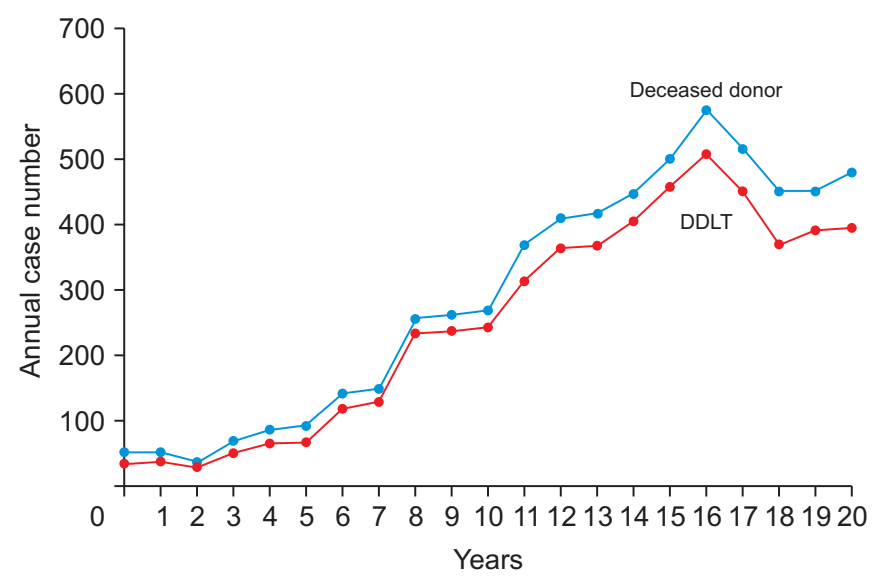

Fig. 1. Nationwide annual number of deceased donors and deceased donor liver transplantation (DDLT) in Korea between the year 2000 and 2020.

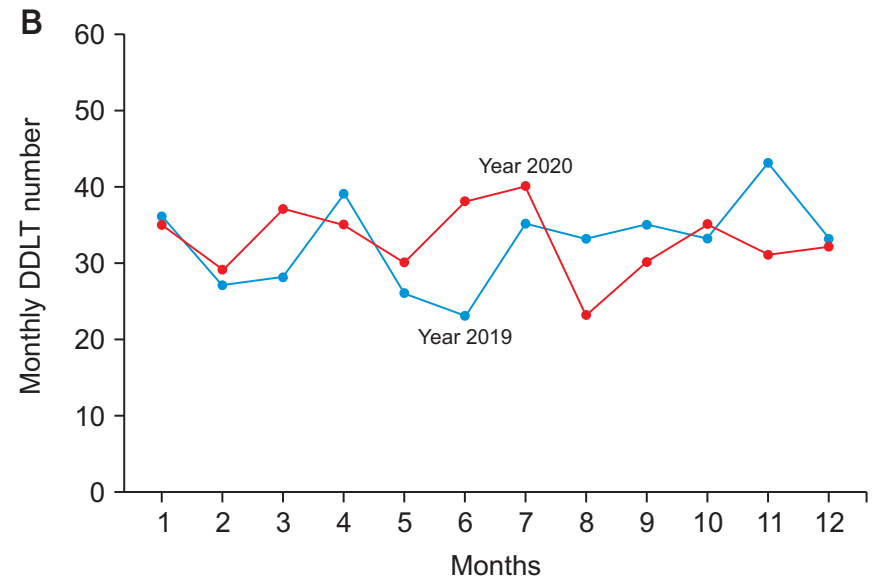

Fig. 2. Comparison of nationwide monthly number of deceased donors (A) and deceased donor liver transplantations (DDLT; B) in Korea between the years 2019 and 2020. 
number of all deceased organ donors was 6,064 and 5,252 cases of DDLTs were performed. The annual number of deceased donors and DDLTs are depicted in Fig. 1.

\section{Comparison of Monthly Number of Deceased Donors and DDLT Operations between the Years 2019 and 2020}

The deceased donor and DDLT case numbers were 450 and 391, respectively, in the year 2019, and 478 and 395 , respectively, in the year 2020 (Fig. 2). The mean number of monthly deceased donors was $37.5 \pm 5.9$ in the year 2019 and $39.8 \pm 4.4$ in the year $2020(p=0.284)$. The mean number of monthly DDLTs was $32.6 \pm 5.7$ in the year 2019 and $32.9 \pm 4.7$ in the year $2020(p=0.877)$. These findings suggest that the COVID-19 pandemic did not significantly affect the number of decreased donors and DDLTs in the year 2020 .

\section{Comparison of Recipient Ages in DDLT and LDLT in the Year 2020}

The numbers of DDLT and LDLT performed in the year 2020 were 395 and 1,221 , respectively. The peak recipient age was 50 years $(n=21)$ in DDLT and 55 years in LDLT $(n=61)$. The distribution of recipient ages according to the types of LT is depicted in Fig. 3. The number of pediatric and adult cases were 17 and 378 , respectively in DDLT, and 32 and 1,189 , respectively, in LDLT $(p=0.090)$. The total number of pediatric LTs was 49 , which constituted $3.0 \%$ of all LT cases.

\section{Comparison of LT Volume in 56 LT Centers in the Year 2020}

A total of 1,616 cases of LT were performed in 56 centers in the year 2020. DDLT and LDLT were performed in 46 and 51 centers, respectively. Five centers performed only DDLT, and their LT volume in the year 2020 was one to two

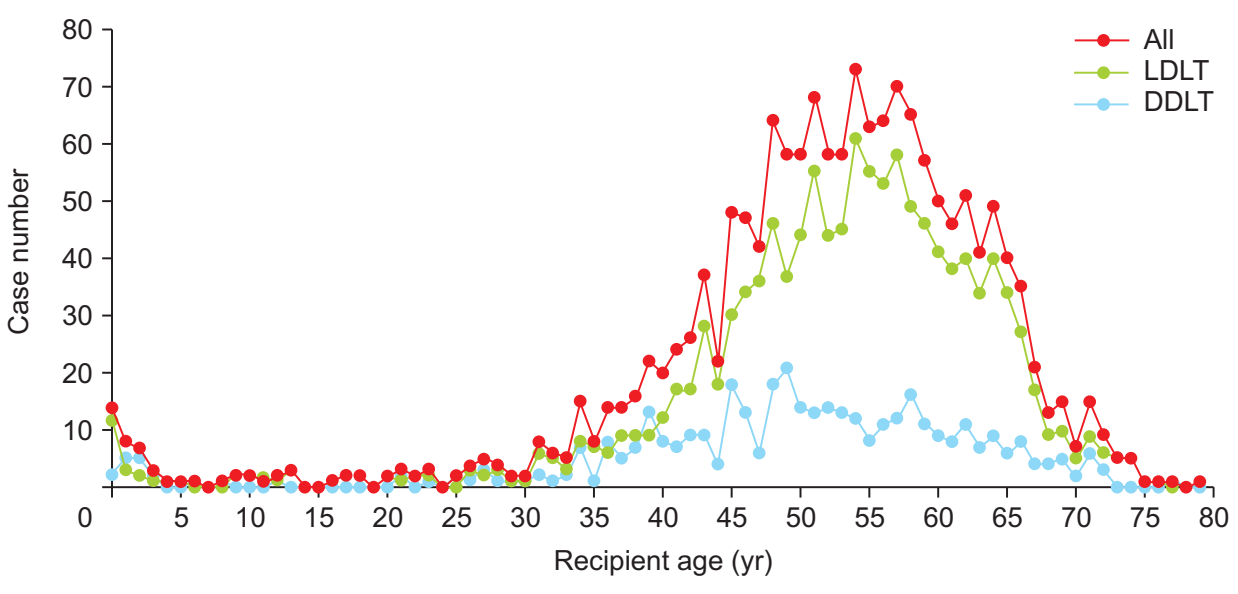

Fig. 3. Distribution of recipient age according to deceased donor liver transplantation (DDLT) and living donor liver transplantation (LDLT) in 2020.

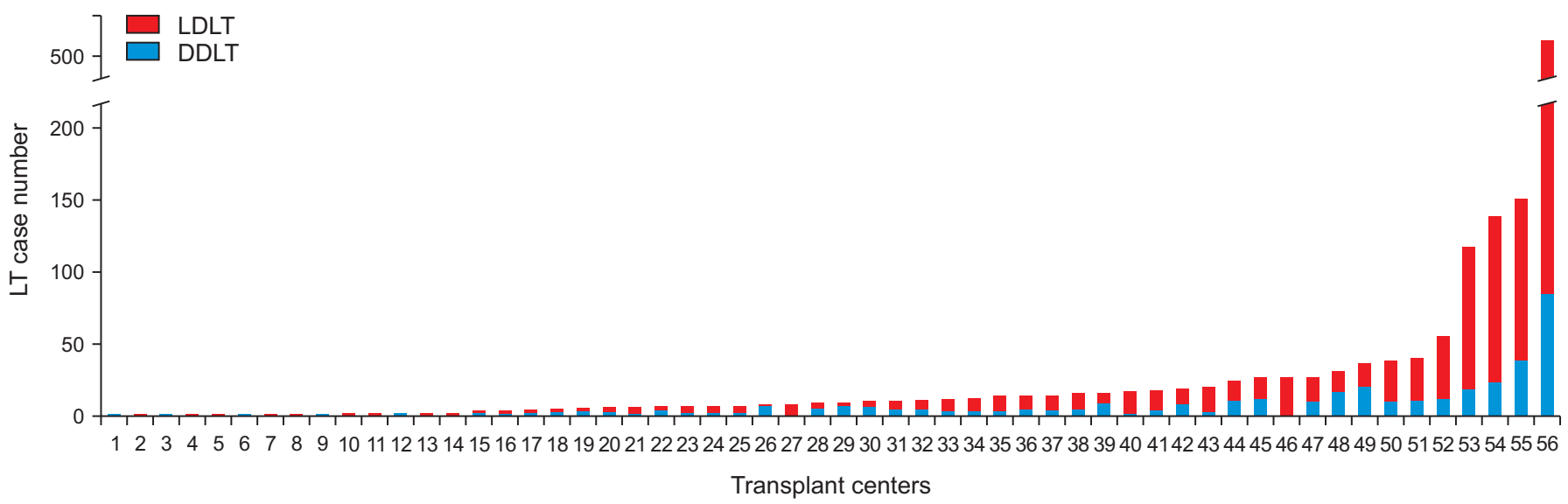

Fig. 4. Distribution of Korean transplantation centers performing deceased donor liver transplantation (DDLT) and living donor liver transplantation (LDLT) in 2020. LT, liver transplantation. 
cases. Another 10 centers performed only LDLT, with an LT volume of one to two cases in 8 centers during the year 2020 , but 8 and 28 cases each were reported at two centers. Forty-one centers performed both DDLT and LDLT (Fig. 4).

In the year 2020, 31 (55.4\%) centers performed 1 to 10 LTs; 13 (23.2\%) centers reported 11 to 25 LTs; 7 (12.5\%) centers performed 26 to $50 \mathrm{LTs}$; one (1.8\%) center conducted 55 LTs; three centers had an LT volume of 118,139 and 151 each; and one (1.8\%) center reported 511 LTs.

The latter five major centers performed 974 LTs, which accounted for $60.3 \%$ of LT volume nationwide in the year 2020. The 511 LTs performed at a single center constituted $31.6 \%$ of the LT volume nationwide.

These major five LT centers performed 179 cases of DDLT, which accounted for $45.3 \%$ of the DDLT volume nationwide; and also performed 795 LDLTs, which constituted $65.1 \%$ of the LDLT volume nationwide $(p<0.001)$. These findings suggest that LDLT was predominantly performed at these high-volume LT centers.

\section{DISCUSSION}

COVID-19 has spread rapidly around the world, prompting the World Health Organization to declare a global pandemic. In the absence of effective treatment for COVID-19, the risk of COVID-19 for patients with end-stage organ disease awaiting transplant surgery is unknown $[5,6]$. As the pandemic worsens around the world, organ procurement from deceased donors has decreased, and LDLT has been postponed to preserve medical resources for COVID-19 and to avoid nosocomial transmission $[7,8]$. During the COVID-19 pandemic, deciding whether or not to proceed with $\mathrm{LT}$ is a challenge. More than one and half years have passed since COVID-19 pandemic, suggesting the need to analyze its real-world effect on LT in Korea.

There are concerns that $\mathrm{LT}$ recipients may be at higher risk of developing severe illness caused by COVID-19 [9]. Although LT recipients might be susceptible to COVID-19 infection, it is uncertain whether immunosuppression alters the clinical features and disease severity of COVID-19. The clinical features of COVID-19 among organ transplant recipients are known to be as heterogeneous as in the general population [10].

Lee et al. [11] analyzed the transplantation activities in Severance Hospital, in which transplantation with living and deceased donors remained stable during the COVID-19 outbreak, compared with the same period in the year 2019.
They also reported that none of their transplant recipients contracted COVID-19.

The results of the present study revealed that national LT activities with living and deceased donors did not decrease in the year 2020, compared with 2019. It suggests that LT is feasible through appropriate infection prevention measures and extensive testing. A Spanish transplant group proposed some general lines of action that require adaptation to LT recipients [12], which is helpful to establish a modification in immunosuppressive treatment before manifestation of any complications.

As shown in the present study, the sudden recent decrease in deceased donor number has yet to be reversed. The annual number of deceased donors temporarily exceeded 10 per million in 2016, but has since decreased, likely because of specific medical and social issues, including the Life Insurance Decision Act regulating termination of life-sustaining treatment [13]. Recently, the results of donation improvement program to increase organ donation at hospitals were reported [14]. This program included education for healthcare professionals of participating hospitals regarding each step of donation as well as evaluation. The authors suggested that this program increases the recognition of brain death and the organ donation rate, and positively affect the attitudes of healthcare professionals toward organ donation, suggesting its promotion across all hospitals to increase organ donation.

The present study revealed that the number of viable LT centers in Korea is as high as 56 . Forty-one transplant centers performed both DDLT and LDLT, but five centers performed only DDLT and another 10 centers conducted only LDLT. The small LT volume in the latter centers suggest a recent implementation of LT program. In contrast, the five major centers performed 974 cases, which accounted for $60.3 \%$ of LT volume nationwide in 2020 . These centers performed $45.3 \%$ of all DDLT volume and $65.1 \%$ of the LDLT volume nationwide in 2020 , indicating that LDLT was highly concentrated at these high-volume LT centers.

This study had several limitations of note. This was a retrospective cohort study with limited information regarding donors and recipients, because detailed data were not available in the KONOS database. Annual follow-up studies are necessary to validate the results of the present study.

In conclusion, the results of this study suggested that the nationwide activities of DDLT and LDLT performed in Korea remained stable in the year 2020, despite the ongoing COVID-19 pandemic. Further studies are necessary to follow up and validate the results of this study. 


\section{FUNDING}

There was no funding related to this study.

\section{CONFLICT OF INTEREST}

All authors have no conflicts of interest to declare.

\section{ORCID}

Yong-Kyu Chung https://orcid.org/0000-0002-2132-2450

Sung-Hwa Kang https://orcid.org/0000-0002-5614-5968

\section{AUTHORS' CONTRIBUTIONS}

Conceptualization: YKC. Data curation: All. Formal analysis: All. Investigation: All. Methodology: All. Resources: YKC. Supervision: YKC. Validation: YKC. Visualization: YKC. Writing - original draft: All. Writing - review \& editing: All.

\section{REFERENCES}

1. Lee SG, Moon DB, Hwang S, Ahn CS, Kim KH, Song GW, et al. Liver transplantation in Korea: past, present, and future. Transplant Proc 2015;47:705-708.

2. Lee JG, Lee KW, Kwon CHD, Chu CW, Kim BW, Choi DL, et al. Donor safety in living donor liver transplantation: the Korean organ transplantation registry study. Liver Transpl 2017;23: 999-1006.

3. Hwang S, Ha TY, Ahn CS, Moon DB, Kim KH, Song GW, et al. Standardized surgical techniques for adult living donor liver transplantation using a modified right lobe graft: a video presentation from bench to reperfusion. Korean J Hepatobiliary Pancreat Surg 2016;20:97-101.

4. Min SI, Ahn C, Han DJ, Kim SI, Chung SY, Lee SK, et al. To achieve national self-sufficiency: recent progresses in deceased donation in Korea. Transplantation 2015;99:765-770.
5. Gori A, Dondossola D, Antonelli B, Mangioni D, Alagna L, Reggiani $\mathrm{P}$, et al. Coronavirus disease 2019 and transplantation: a view from the inside. Am J Transplant 2020;20:1939-1940.

6. Michaels MG, La Hoz RM, Danziger-Isakov L, Blumberg EA, Kumar D, Green M, et al. Coronavirus disease 2019: implications of emerging infections for transplantation. Am J Transplant 2020;20:1768-1772.

7. Angelico R, Trapani S, Manzia TM, Lombardini L, Tisone G, Cardillo M. The COVID-19 outbreak in Italy: initial implications for organ transplantation programs. Am J Transplant 2020; 20:1780-1784.

8. Boyarsky BJ, Po-Yu Chiang T, Werbel WA, Durand CM, Avery RK, Getsin SN, et al. Early impact of COVID-19 on transplant center practices and policies in the United States. Am J Transplant 2020;20:1809-1818.

9. Kumar D, Manuel O, Natori Y, Egawa H, Grossi P, Han SH, et al. COVID-19: a global transplant perspective on successfully navigating a pandemic. Am J Transplant 2020;20:1773-1779.

10. Columbia University Kidney Transplant Program. Early description of coronavirus 2019 disease in kidney transplant recipients in New York. J Am Soc Nephrol 2020;31:1150-1156.

11. Lee J, Kim EJ, Ihn K, Lee JG, Joo DJ, Kim MS, et al. The feasibility of organ transplantation during the COVID-19 outbreak: experiences from South Korea. Korean J Transplant 2020;34: 257-264.

12. Forns $X$, Navasa M. Liver transplant immunosuppression during the COVID-19 pandemic. Gastroenterol Hepatol 2020; 43:457-463.

13. Ha HS, Hong JJ, Kim IO, Lee SR, Lee AY, Ha TY, et al. Deceased donor liver transplantation under the Korean model for end-stage liver disease score-based liver allocation system: 2-year allocation results at a high-volume transplantation center. Korean J Transplant 2019;33:112-117.

14. Heo SJ, Ju YH, Noh EJ, Kim KM, Son YK, Jung SW, et al. A study on the performance of the donation improvement program in Korea. Korean J Transplant 2021;35:77-85. 